\title{
Gneiting Class, Semi-Metric Spaces and Isometric Embeddings
}

\author{
VAldir A. Menegatto*, Claudemir P. Oliveira And Emilio PorCu
}

\begin{abstract}
This paper revisits the Gneiting class of positive definite kernels originally proposed as a class of covariance functions for space-time processes. Under the framework of quasi-metric spaces and isometric embeddings, the paper proposes a general and unifying framework that encompasses results provided by earlier literature. Our results allow to study the positive definiteness of the Gneiting class over products of either Euclidean spaces or high dimensional spheres and quasi-metric spaces. In turn, Gneiting's theorem is proved here by a direct construction, eluding Fourier inversion (the so-called Gneiting's lemma) and convergence arguments that are required by Gneiting to preserve an integrability assumption.
\end{abstract}

Keywords: Gneiting class, positive definite, completely monotone, isometric embedding, quasi-metric spaces.

2010 Mathematics Subject Classification: 42A82, 43A35.

\section{INTRODUCTION}

Positive definite kernels have a long history that traces back to many branches of pure and applied mathematics, as well as to statistics, machine learning, computer science and other applied sciences. Positive definite and radially symmetric kernels on metric spaces have been introduced in the seminal papers $[22,23]$.

There has been a growing interest in the last twenty years for positive definiteness over product spaces. The main motivation stems from stochastic processes defined continuously over subsets of the type $X \times Y$, where $X$ is a subset of the $d$-dimensional Euclidean space $\mathbb{R}^{d}$, and $Y$ is either the whole real line or the set of integers $\mathbb{Z}$, and represents time. The nomenclature space-time covariance functions is commonly accepted for kernels that are positive definite over such product spaces, and the reader is referred to [8] for a review. A wealth of literature is available for the case $X=\mathbb{R}^{d}$, and the reader is referred to $[2,7,16,18,21]$ and to [6] for relevant contributions. Recently, much attention has been put on the case $X=S^{d-1}$, the unit sphere embedded in $\mathbb{R}^{d}$. A characterization theorem for this case (including the Hilbert sphere $S^{\infty}$ ) is available thanks to [4]. Other contributions can be found in [10,15] and recently in [27].

This paper considers quasi-metric spaces, that is, pairs $(X, \sigma)$ where $X$ is a non-empty set and $\sigma$ is a quasi-distance, that is, a function $\sigma: X \times X \rightarrow[0, \infty)$ satisfying $\sigma\left(x, x^{\prime}\right)=\sigma\left(x^{\prime}, x\right)$, $x, x^{\prime} \in X$, and $\sigma(x, x)=0, x \in X$. A semi-metric space $(X, \sigma)$ is a quasi-metric space if in addition to the previous properties one has $\sigma$ satisfying the triangle inequality. Further, if $\sigma\left(x, x^{\prime}\right)>0$ when $x \neq x^{\prime}$, the semi-metric space $(X, \sigma)$ becomes a metric space.

Normed spaces and inner product spaces are typical examples of quasi-metric spaces with quasi-distance given by $\sigma\left(x, x^{\prime}\right)=\left\|x-x^{\prime}\right\|, x, x^{\prime} \in X$, where $\|\cdot\|$ is the norm of the space. The notion of semi-metric spaces is usually preferred when one deals with isometric embeddings.

Received: 31.03.2020; Accepted: 15.05.2020; Published Online: 16.05.2020

*Corresponding author: V. A. Menegatto; menegatt@icmc.usp.br

DOI: $10.33205 / \mathrm{cma} .712049$ 
A quasi-metric space $(X, \sigma)$ is isometrically embeddable in a Hilbert space $(\mathbb{H},\langle\cdot, \cdot\rangle)$ if there exists a mapping $i: X \rightarrow \mathbb{H}$ such that

$$
\left\langle i(x)-i\left(x^{\prime}\right), i(x)-i\left(x^{\prime}\right)\right\rangle=\sigma\left(x, x^{\prime}\right)^{2}, \quad x, x^{\prime} \in X .
$$

This notion is explored in [28] and discussed in [3].

Let $E$ be a nonempty set. A mapping $\varphi: E \times E \rightarrow \mathbb{R}$ is called positive definite if

$$
\sum_{k, l=1}^{N} c_{k} c_{l} \varphi\left(x_{k}, x_{l}\right) \geq 0,
$$

for any collection $\left\{c_{k}: k=1, \ldots, N\right\} \subset \mathbb{R}$ and any $\left\{x_{1}, \ldots, x_{k}\right\} \subset E$. If $E$ is a quasi-metric space $(X, \sigma)$, the positive definite function $\varphi$ on $E$ is usually demanded to be metric-dependent in the sense that

$$
\varphi\left(x, x^{\prime}\right)=f\left(\sigma\left(x, x^{\prime}\right)\right), \quad x, x^{\prime} \in X,
$$

where $f$ is a continuous function. Obviously, the domain of $f$ is understood to be the diameter set of $X$, that is,

$$
D_{X}^{\sigma}=\left\{\sigma\left(x, x^{\prime}\right): x, x^{\prime} \in X\right\},
$$

while continuity on a semi-metric space is defined the same way it is so in a metric-space. Depending on $E$ and its metric structure, one may find convenient characterizations for the positive definiteness of a function on $E$. One case that is somehow related to the present work involves the case where $E=\mathbb{R}^{d}$ without any metric structure but the function $\varphi$ being translation-invariant, that is,

$$
\varphi(x, y)=f(x-y), \quad x, y \in \mathbb{R}^{d},
$$

for some continuous function $f: \mathbb{R}^{d} \rightarrow \mathbb{R}$. In this case, a result of Bochner ([1]) shows that $\varphi$ is positive definite if and only if $f$ is the Fourier transform of a finite and positive Borel measure $\mu$, i.e.,

$$
f(x)=\int_{\mathbb{R}^{d}} e^{i x \cdot w} d \mu(w), \quad x \in \mathbb{R}^{d},
$$

with $\cdot$ denoting the dot product in $\mathbb{R}^{d}$.

For two quasi-metric spaces $(X, \sigma)$ and $(Y, \nu)$, we denote by $P D(X \times Y, \sigma, \nu)$ the class of continuous functions $\varphi: D_{X}^{\sigma} \times D_{Y}^{\nu} \rightarrow \mathbb{R}$ such that the composite kernel

$$
\left((x, y),\left(x^{\prime}, y^{\prime}\right)\right) \mapsto \varphi\left(\sigma\left(x, x^{\prime}\right), \nu\left(y, y^{\prime}\right)\right), \quad(x, y),\left(x^{\prime}, y^{\prime}\right) \in X \times Y,
$$

is positive definite on $X \times Y$. Analogously, we write $P D(X, \sigma)$ for the class of functions $\varphi$ : $D_{X}^{\sigma} \rightarrow \mathbb{R}$ being continuous and such that the kernel $\left(x, x^{\prime}\right) \mapsto \varphi\left(\sigma\left(x, x^{\prime}\right)\right)$ is positive definite.

Next, let us recall the notion of complete monotonicity. A function $f:(0, \infty) \rightarrow \mathbb{R}$ is called completely monotone if it is infinitely often differentiable over $(0, \infty)$ and $(-1)^{n} f^{(n)}(t) \geq 0$ for all $t>0$ and all $n=0,1, \ldots$ In this paper, we will assume all completely monotone functions are bounded so that they have a continuous extension to $[0, \infty)$. In particular, $f(0)<\infty$. A nonnegative function $f:(0, \infty) \rightarrow \mathbb{R}$ having a completely monotone derivative is called a Bernstein function. A Bernstein function can be continuously extended to $[0, \infty)$. Additional information on completely monotone and Bernstein functions can be found in [20].

This paper deals with a class $\left\{G_{\alpha}: \alpha>0\right\}$ of continuous functions, where

$$
G_{\alpha}(t, u)=\frac{1}{h(u)^{\alpha}} f\left(\frac{t}{h(u)}\right), \quad t, u \geq 0,
$$

with $f$ and $h$ strictly positive and continuous. In principle, both functions $f$ and $h$ are defined over $[0, \infty)$, but they might be restricted to suitable subsets of $[0, \infty)$. Such a class has been 
especially popular in space-time geostatistics for the following reason: for $d$ and $l$ positive integers, $f$ being bounded and completely monotone and $h$ such that $\exp (-c h) \in P D\left(\mathbb{R}^{l},\|\cdot\|\right)$ for all $c>0$, where $\|\cdot\|$ stands for the Euclidean norm, sufficient conditions for $G_{\alpha}$ to belong to the class $P D\left(\mathbb{R}^{d} \times \mathbb{R}^{\ell},\|\cdot\|^{2},\|\cdot\|^{2}\right)$ were given in [7]. The resulting class $\left\{G_{\alpha}: \alpha \geq d / 2\right\}$ is usually called Gneiting's class. For $f$ being bounded completely monotone, necessary and sufficient conditions on $h$ have been provided by [30] in order that $G_{\alpha}$ belong to $P D\left(\mathbb{R}^{d} \times Y,\|\cdot\|^{2},\|\cdot\|_{Y}\right)$, where $\left(Y,\|\cdot\|_{Y}\right)$ is a normed linear space. Porcu et al. ([15]) presented sufficient conditions for $G_{\alpha}$ to belong to the class $P D\left(\mathbb{R}^{d} \times S^{m},\|\cdot\|^{2}, \theta_{m}\right)$, where $\theta_{m}$ is the geodesic distance over $S^{m}$. Sufficient conditions for $G_{\alpha}$ to belong to the class $P D\left(S^{m} \times \mathbb{R}^{d}, \theta_{m},\|\cdot\|^{2}\right)$ for all $d$ and $m$ were shown recently in [27]. In [21], Schlather has considered diagonalized versions $\tilde{G}_{\alpha}(t):=$ $G_{\alpha}(t, t), t \geq 0$, of $G_{\alpha}$. Minor modifications of $G_{\alpha}$ within the class $P D\left(\mathbb{R}^{d} \times \mathbb{R},\|\cdot\|^{2},|\cdot|\right)$ have been proposed by [6] and [17]. Finally, [16] has considered the class $P D\left(\prod_{k=1}^{m} \mathbb{R}^{k},\|\cdot\|^{2}, \ldots,\|\cdot\|^{2}\right)$ on the basis of a generalization of the function $G_{\alpha}$.

The previous paragraph cannot be detached from a classical result proved by I. J. Schoenberg ([23]) involving conditionally negative definite functions. Recall that for a quasi-metric space $(X, \sigma)$, a continuous function $f: D_{X}^{\sigma} \rightarrow \mathbb{R}$ is conditionally negative definite on $X$, and we write $f \in C N D(X, \sigma)$, if for $n \geq 1$ and points $x_{1}, \ldots, x_{N}$ in $X$, it holds

$$
\sum_{j, k=1}^{N} c_{j} c_{k} f\left(\sigma\left(x_{j}, x_{k}\right)\right) \leq 0,
$$

for all real numbers $c_{1}, \ldots, c_{n}$ satisfying $\sum_{j=1}^{N} c_{j}=0$. If $(X, \sigma)$ is a quasi-metric space, a function $h: D_{X}^{\sigma} \rightarrow \mathbb{R}$ belongs to $C N D(X, \sigma)$ if and only if all the functions $u \in D_{X}^{\sigma} \mapsto \exp (-\operatorname{sh}(u))$, $s>0$, belong to $P D(X, \sigma)$. In particular, some of results described in the previous paragraph can be re-established with the CND nomenclature.

Given the existing results, it is natural to ask for results that allow for a very general version as well as for a unifying framework. The plan of this paper is the following. Section 2 provides the necessary background, some preliminary results, a general abstract result that produces functions in $P D\left(\mathbb{R}^{d} \times X,\|\cdot\|, \sigma\right)$, where $(X, \sigma)$ is quasi-metric and examples. In particular, the results imply an alternative proof of the original Gneiting's result that does not involve convergence arguments. Section 3 contains expanded versions of Gneiting's result and adaptations to the case, where one of the spaces is $\left(S^{m}, \theta_{m}\right)$.

\section{PRELIMINARY FINDINGS}

Positive definite functions of the type (1.1) that are additionally radially symmetric are characterized as those functions $f$ belonging to the class $P D\left(\mathbb{R}^{d},\|\cdot\|\right)$. Define the function $\Omega_{d}:[0, \infty) \rightarrow \mathbb{R}$ through $\Omega_{d}(0)=1$ and the identity

$$
\Omega_{d}(t)=\Gamma(d / 2)\left(\frac{2}{t}\right)^{(d-2) / 2} J_{(d-2) / 2}(t), \quad t>0,
$$

where $J_{\nu}$ is the Bessel function of first kind and order $\nu$. As showed in [23], the continuous function $f:[0, \infty) \rightarrow \mathbb{R}$ with $f(0)=1$ belongs to the class $P D\left(\mathbb{R}^{d},\|\cdot\|\right)$ if and only if

$$
f(t)=\int_{[0, \infty)} \Omega_{d}(r t) d \mu(r), \quad t \geq 0,
$$

where $\mu$ is a probability measure. Arguments in [5] show that $\Omega_{d}$ is the characteristic function of a random vector being uniformly distributed over the unit spherical shell $S^{d-1}$ embedded 
in $\mathbb{R}^{d}$. Also, a convergence argument in [23] reveals that the class $\bigcap_{d} P D\left(\mathbb{R}^{d},\|\cdot\|\right)$ is uniquely determined through scale mixtures of the type

$$
f(t)=\int_{[0, \infty)} e^{-r t^{2}} d \mu(r), \quad t \geq 0,
$$

with $\mu$ as before. This fact has a striking connection with completely monotone functions. By Bernstein-Widder's theorem ([26]), a continuous function $f:[0, \infty) \rightarrow \mathbb{R}$ restricts to a completely monotone if and only if it is the Laplace transform of a positive and bounded measure $\mu$ :

$$
f(t)=\int_{[0, \infty)} e^{-r t} d \mu(r), \quad t \geq 0 .
$$

In particular, this shows that $f \in P D\left(\mathbb{R}^{d},\|\cdot\|\right)$ if and only if $t \in(0, \infty) \mapsto f(\sqrt{t})$ is completely monotone.

For $d$ a positive integer, let $L_{d-1}^{1}$ denote the class of real measurable functions $g$ on $[0, \infty)$ for which $\int_{0}^{\infty}|g(r)| r^{d-1} d r<\infty$. The Fourier-Bessel transform $\mathcal{F}_{d}(g)$ of order $(d-2) / 2$ of a function $g \in L_{d-1}^{1}$ is defined by

$$
\mathcal{F}_{d}(g)(t)=\int_{0}^{\infty} g(r) \Omega_{d}(t r) r^{d-1} d r, \quad t \in[0, \infty) .
$$

It is well-known that $\mathcal{F}_{d}$ maps continuously and injectively $L_{d-1}^{1}$ into the set $C_{0}([0, \infty))$ of continuous functions on $[0, \infty)$ vanishing at infinity ([25, chapter 5]). On the other hand, the fact that $t \in[0, \infty) \mapsto \Omega_{d}(t r), r>0$, belongs to $P D\left(\mathbb{R}^{d},\|\cdot\|\right)$, implies that the following elementary result holds.

Proposition 2.1. If $g:[0, \infty) \rightarrow[0, \infty)$ belongs to $L_{d-1}^{1}$, then $\mathcal{F}_{d}(g)$ belongs to $P D\left(\mathbb{R}^{d},\|\cdot\|\right)$.

A generalization of Proposition 2.1 is stated below and will turn to be very useful for the findings following subsequently.

Theorem 2.2. Let $(X, \sigma)$ be a quasi-metric space. Let $g:[0, \infty) \times D_{X}^{\sigma} \rightarrow \mathbb{R}$ satisfy the following assertions:

(i) $g(\cdot, u)$ belongs to $L_{d-1}^{1}$ for any fixed $u \in D_{X}^{\sigma}$;

(ii) $g(r, \cdot)$ belongs to $P D(X, \sigma)$ for any fixed $r \geq 0$.

If the mapping $(t, u) \in[0, \infty) \times D_{X}^{\sigma} \mapsto \mathcal{F}_{d}(g(\cdot, u))(t)$ is continuous on $[0, \infty) \times D_{X}^{\sigma}$, then it belongs to the class $P D\left(\mathbb{R}^{d} \times X,\|\cdot\|, \sigma\right)$.

Proof. Using Equation (2.5), the function $\mathcal{F}_{d}(g(\cdot, u))$ can be written as

$$
\mathcal{F}_{d}(g(\cdot, u))(t)=\int_{0}^{\infty} g(r, u) \Omega_{d}(t r) r^{d-1} d r, \quad(t, u) \in[0, \infty) \times D_{X}^{\sigma},
$$

which, in concert with Schur product theorem ([11, p. 455]), completes the proof. [14].

An implication of Bernstein-Widder's theorem is stated below. More details can be found in

Proposition 2.3. Let $(X, \sigma)$ be a quasi-metric space. If $f$ is bounded and completely monotone and $h$ is a nonnegative valued function in $C N D(X, \sigma)$, then $f \circ h$ belongs to $P D(X, \sigma)$.

Proposition 2.3 is very useful to discuss the following important example. 
Example 2.4. Let $(X, \sigma)$ be a quasi-metric space and $d$ a positive integer. Let $h$ be a nonnegative valued function in $C N D(X, \sigma)$. Then, we claim that

$$
(t, u) \in[0, \infty) \times D_{Y}^{\sigma} \mapsto \frac{e^{-t \sqrt{h(u)}}}{\sqrt{h(u)}}
$$

belongs to $P D\left(\mathbb{R}^{d} \times X,\|\cdot\|, \sigma\right)$. To show it, let $v>0$. We first recall the identity $([9$, p. 678])

$$
\int_{0}^{\infty} r^{d / 2}\left(r^{2}+v^{2}\right)^{-(d+1) / 2} J_{(d-2) / 2}(t r) d r=\frac{\sqrt{\pi} t^{(d-2) / 2}}{2^{d / 2} v e^{v t} \Gamma((d+1) / 2)}, \quad v, t>0 .
$$

Resorting to Equation (2.3), and rearranging terms, we obtain

$$
\int_{0}^{\infty}\left(r^{2}+v^{2}\right)^{-(d+1) / 2} \Omega_{d}(t r) r^{d-1} d r=\frac{\Gamma(d / 2) \sqrt{\pi}}{2 v e^{v t} \Gamma((d+1) / 2)}, \quad v, t>0 .
$$

Since the function on the right hand side is continuous, by letting $t \rightarrow 0^{+}$we have that the identity above holds for $t=0$ as well. Since, for $r$ fixed, $v \in(0, \infty) \mapsto\left(r^{2}+v\right)^{-(d+1) / 2}$ is bounded and completely monotone, if $h$ is a nonnegative valued function in $(X, \sigma)$, then Proposition 2.3 shows that $u \in D_{X}^{\sigma} \mapsto\left(r^{2}+h(u)\right)^{-(d+1) / 2}$ belongs to $P D(X, \sigma)$. After ignoring positive constants, we can invoke Theorem 2.2 to show our claim.

It might be interesting to note that this example does not belong to the Gneiting class $G_{\alpha}$.

We now rephrase Theorem 2.2 according to the language of Fourier transforms. For an absolutely integrable function $F$ in $\mathbb{R}^{d}$, its Fourier transform $\widehat{F}$ is given by the formula

$$
\widehat{F}(x)=\frac{1}{(2 \pi)^{d / 2}} \int_{\mathbb{R}^{d}} F(y) e^{-i x \cdot y} d y, \quad x \in \mathbb{R}^{d} .
$$

It is well known that if $F$ is radial, that is, $F(x)=f(\|x\|)$, for some function $f:[0, \infty) \rightarrow \mathbb{R}$, then $\widehat{F}$ is radial as well [5]. For a function $G: \mathbb{R}^{d} \times D_{X}^{\sigma} \rightarrow \mathbb{R}$, we write $\widehat{G}(\cdot, u)$ to denote the Fourier transform of $x \in \mathbb{R}^{d} \mapsto G(x, u)$, for a fixed $u$, whenever it exists. If $G(\cdot, u)$ is radial in the first variable, that is,

$$
G(x, u)=g(\|x\|, u), \quad x \in \mathbb{R}^{d},
$$

for some $g$ and $\widehat{G}(\cdot, u)$ exists, then we may also write $\widehat{G}(x, u)=\widehat{g}(\|x\|, u)$, for some function $\widehat{g}(\cdot, u)$. This notation appears below.

Theorem 2.5. Let $(X, \sigma)$ be a quasi-metric space. Let $G: \mathbb{R}^{d} \times D_{X}^{\sigma} \rightarrow \mathbb{R}$ be radial in the first variable and assume the following assumptions hold:

(i) $g(\cdot, u)$ belongs to $L_{d-1}^{1}$ for any fixed $u \in D_{X}^{\sigma}$;

(ii) $g(r, \cdot)$ belongs to $P D(X, \sigma)$ for any fixed $r \geq 0$.

If the mapping $(t, u) \in[0, \infty) \times D_{X}^{\sigma} \mapsto \widehat{g}(t, u)$ is continuous on $[0, \infty) \times D_{X}^{\sigma}$, then it belongs to the class $P D\left(\mathbb{R}^{d} \times X,\|\cdot\|, \sigma\right)$.

Proof. Theorem 5.26 in [29] shows that if $g(\cdot, u) \in L_{d-1}^{1}$ for any $u \in D_{X}^{\sigma}$, then $g(\|\cdot\|, u)$ is absolutely integrable in $\mathbb{R}^{d}$. In particular, $\widehat{G}(\cdot, u)$ is well defined for any fixed $u$. Invoking again Theorem 5.26 in [29], we have that

$$
\widehat{G}(x, u)=\frac{2^{-(d-2) / 2}}{\Gamma(d / 2)} \mathcal{F}_{d}(g(\cdot, u))(\|x\|), \quad x \in \mathbb{R}^{d} ; u \in D_{X}^{\sigma} .
$$

An application of Theorem 2.2 completes the proof.

An illustration of Theorem 2.5 follows. 
Proposition 2.6. Let $(X, \sigma)$ be a quasi-metric space and $h$ a nonnegative valued function in the class $C N D(X, \sigma)$. For $s>0$, let $H_{s}$ be the function defined through

$$
H_{s}(t, u)=\frac{e^{-s t^{2} / h(u)}}{h(u)^{d / 2}}, \quad(t, u) \in[0, \infty) \times D_{X}^{\sigma} .
$$

Then $H_{s}$ belongs to $P D\left(\mathbb{R}^{d} \times X,\|\cdot\|, \sigma\right)$ for all $s$.

Proof. We start by invoking the well-known identity ([1, p.13])

$$
e^{-\|v\|^{2} / 2}=\frac{1}{(2 \pi)^{d / 2}} \int_{\mathbb{R}^{d}} e^{-i v \cdot w} e^{-\|w\|^{2} / 2} d w, \quad v \in \mathbb{R}^{d} .
$$

Elementary Fourier inversion allows to write

$$
2^{d / 2} \frac{e^{-\|v\|^{2} / \xi h(u)}}{\xi^{d / 2} h(u)^{d / 2}}=\frac{1}{(2 \pi)^{d / 2}} \int_{\mathbb{R}^{d}} e^{-i v \cdot w} G(\|w\|, u) d w, \quad v \in \mathbb{R}^{d} ; \xi>0,
$$

where $G:[0, \infty) \times D_{X}^{\sigma} \rightarrow \mathbb{R}$ is given through the identity

$$
G(x, u)=e^{-s h(u) x^{2} / 4}, \quad x \geq 0 ; u \in D_{X}^{\sigma} .
$$

Since $G$ is radial in the first argument, we can now write

$$
g(r, u)=e^{-s h(u) r^{2} / 4}, \quad r \geq 0 ; u \in D_{X}^{\sigma} .
$$

Proposition 2.3 shows that $g(r, \cdot)$ satisfies Assumption (ii) in Theorem 2.5 for all $r \geq 0$. Assumptions $(i)$ holds trivially, while for $\xi>0$ fixed, the function

$$
\widehat{g}(t, u)=2^{d / 2} \frac{e^{-t^{2} / \xi h(u)}}{\xi^{d / 2} h(u)^{d / 2}}, \quad(t, u) \in[0, \infty) \times D_{X}^{\sigma},
$$

is continuous. Theorem 2.5 shows that

$$
(t, u) \in[0, \infty) \times D_{X}^{\sigma} \mapsto 2^{d / 2} \frac{e^{-t^{2} / \xi h(u)}}{\xi^{d / 2} h(u)^{d / 2}},
$$

belongs to the class $P D\left(\mathbb{R}^{d} \times X,\|\cdot\|, \sigma\right)$ for all positive $\xi$. A change of variable of the type $\xi=1 / s$ and the fact that we can ignore multiplicative positive constants complete the proof.

Remark 2.7. The function $H_{s}$ provides a way to prove Gneiting's theorem [7] by direct construction, without resorting to Fourier transform techniques which in turn require integrability assumptions and the application of a convergence argument.

Remark 2.8. Proposition 2.6 can be also proved by invoking Theorem 2.2 , in concert with the identity

$$
\int_{0}^{\infty} e^{-h(u) r^{2} / 4 s} \Omega_{d}(t r) r^{d-1} d r=2^{d-1} s^{d / 2} \Gamma(d / 2) \frac{e^{-s t^{2} / h(u)}}{h(u)^{d / 2}}, \quad s>0 ; t \geq 0 ; u \in D_{X}^{\sigma},
$$

that is derived from the equality (see $[9$, p.706])

$$
\int_{0}^{\infty} r^{d / 2} e^{-v r^{2}} J_{(q-2) / 2}(t r) d r=t^{(d-2) / 2} \frac{e^{-t^{2} / 4 v}}{(2 v)^{q / 2}}, \quad t, v>0 .
$$


Example 2.9. We use Formula 69 in Chapter 25 of [19]:

$$
\int_{0}^{\infty} r^{(d+4) / 2} e^{-v r^{2}} J_{(d-2) / 2}(t r) d r=\frac{t^{(d-2) / 2}}{2^{d / 2} v^{(d+2) / 2}}\left(\frac{d}{2}-\frac{t^{2}}{4 v}\right) e^{-t^{2} / 4 v}, \quad t, v>0 .
$$

Simple algebra manipulation as in the previous example leads to

$$
\int_{0}^{\infty} r^{2} e^{-v r^{2}} \Omega_{d}(t r) r^{d-1} d r=\frac{\Gamma(d / 2)}{2}\left(\frac{d}{2}-\frac{t^{2}}{4 v}\right) \frac{e^{-t^{2} / 4 v}}{v^{1+d / 2}}, \quad v>0 ; t \geq 0 .
$$

Replacing $v$ by $h(u) /(4 s)$, with $h \in C N D(X, \sigma)$, yields that

$$
\int_{0}^{\infty} r^{2} e^{-h(u) r^{2} / 4 s} \Omega_{d}(t r) r^{d-1} d r=\Gamma(d / 2) 2^{d+1} s^{1+d / 2}\left(\frac{d}{2}-\frac{s t^{2}}{h(u)}\right) \frac{e^{-s t^{2} / h(u)}}{h(u)^{1+d / 2}},
$$

for $s, t>0$ and $u \in D_{X}^{\sigma}$. Theorem 2.2 now shows that

$$
(t, u) \in[0, \infty) \times D_{X}^{\sigma} \mapsto s^{1+d / 2}\left(\frac{d}{2}-\frac{s t^{2}}{h(u)}\right) \frac{e^{-s t^{2} / h(u)}}{h(u)^{1+d / 2}}
$$

belongs to $P D\left(\mathbb{R}^{d} \times X,\|\cdot\|, \sigma\right)$. We can also integrate with respect to $s$ in order to see that

$$
(t, u) \in[0, \infty) \times D_{X}^{\sigma} \mapsto \frac{1}{h(u)^{1+d / 2}} \int_{0}^{\infty} s^{1+d / 2}\left(\frac{d}{2}-\frac{s t^{2}}{h(u)}\right) e^{-s t^{2} / h(u)} d \mu(s)
$$

also belongs to $P D\left(\mathbb{R}^{d} \times X,\|\cdot\|, \sigma\right)$ as longs as $\mu$ is a convenient measure on $[0, \infty)$. Again, this example does not belong to the Gneiting class $G_{\alpha}$.

\section{Gneiting Class: Results}

The following result is another implication of Bernstein-Widder's theorem. As in Proposition 2.3, it can be extracted from [14].

Proposition 3.1. Let $(X, \sigma)$ be a quasi-metric space. Let $g$ be a Bernstein function and $h$ a nonnegative valued function in $C N D(X, \sigma)$. Then, $\exp (-c(g \circ h))$ belongs to $P D(X, \sigma)$ for all $c>0$.

We are now ready to state and prove one of our main contributions.

Theorem 3.2. Let $d$ be a positive integer and $(X, \sigma)$ a quasi-metric space. Let $G_{\alpha}$ be the function defined at Equation (1.2) with $f$ being completely monotone. If $a \in(0,1]$ and $\alpha \geq d / 2$, then the following assertions are true:

(i) $G_{\alpha}$ belongs to $P D\left(\mathbb{R}^{d} \times X,\|\cdot\|^{2 a}, \sigma\right)$ provided $h$ is a nonnegative valued function in the class $C N D(X, \sigma)$;

(ii) $G_{\alpha}$ belongs to $P D\left(\mathbb{R}^{d} \times X,\|\cdot\|^{2 a}, \sigma\right)$ provided $h:=g \circ h_{1}$, where $g$ is a positive Bernstein function and $h_{1}$ is a nonnegative function in $C N D(X, \sigma)$;

(iii) $G_{\alpha}$ belongs to $P D\left(\mathbb{R}^{d} \times X,\|\cdot\|^{2 a}, \sigma^{2 b}\right)$ provided $b \in(0,1], h$ is a positive Bernstein function, and $(X, \sigma)$ is isometrically embeddable in a Hilbert space.

Proof. Let us show Assertion $(i)$ by invoking Proposition 2.6, which shows that $H_{s}$ in Equation (2.6) defines an element of the class $P D\left(\mathbb{R}^{d} \times X,\|\cdot\|, \sigma\right)$ for all $s>0$. This in turn shows, in concert with Bernstein-Widder's theorem that

$$
G_{d / 2}\left(t^{2}, u\right)=\frac{1}{h(u)^{d / 2}} \int_{0}^{\infty} e^{-s t^{2} / h(u)} d \mu(s)=\int_{0}^{\infty} H_{s}(t, u) d \mu(s)
$$


is also a member of $P D\left(\mathbb{R}^{d} \times X,\|\cdot\|, \sigma\right)$, and thus $G_{d / 2}(t, u) \in P D\left(\mathbb{R}^{d} \times X,\|\cdot\|^{2}, \sigma\right)$. We now observe that if $h$ is a nonnegative valued function in $C N D(X, \sigma)$, then the Laplace transform identity

$$
\frac{1}{x}=\int_{0}^{\infty} e^{-s x} d s, \quad x>0,
$$

shows that $1 / h \in P D(X, \sigma)$, while the identity

$$
x^{a}=\left(\int_{0}^{\infty} \frac{1-e^{-s^{2}}}{s^{1+2 a}} d s\right)^{-1} \int_{0}^{\infty} \frac{1-e^{-s^{2} x}}{s^{1+2 a}} d s, \quad x \geq 0 ; a \in(0,1),
$$

implies that $h^{a} \in P D(X, \sigma)$ for $a \in(0,1)$. To proceed, for $\alpha \geq d / 2$, write

$$
G_{\alpha}(t, u)=\frac{1}{h(u)^{\alpha-d / 2}} G_{d / 2}(t, u), \quad t, u \geq 0,
$$

and notice that Proposition 2.3 shows that

$$
u \in[0, \infty) \mapsto \frac{1}{h(u)^{\alpha-d / 2}}
$$

belongs to $P D(X, \sigma)$. Since it is an easy matter to verify that

$$
(t, u) \in[0, \infty)^{2} \mapsto \frac{1}{h(u)^{\alpha-d / 2}}
$$

belongs to $P D\left(\mathbb{R}^{d} \times X,\|\cdot\|, \sigma\right)$, we may invoke the Schur product theorem in order to deduce that $G_{\alpha} \in P D\left(\mathbb{R}^{d} \times X,\|\cdot\|^{2}, \sigma\right)$, for $\alpha \geq d / 2$. Finally, for any Hilbert space $\mathbb{H}$, it is wellknown that the semi-metric space $\left(\mathbb{H},\|\cdot\|^{a}\right)$ is isometrically embeddable into $(\mathbb{H},\|\cdot\|)$ itself. Therefore, we have that $G_{\alpha} \in P D\left(\mathbb{R}^{d} \times X,\|\cdot\|^{2 a}, \sigma\right)$. Assertion $(i i)$ follows from $(i)$ in concert with Proposition 3.1. If $(X, \sigma)$ is isometrically embeddable in a Hilbert space, then the function $h_{1}(u)=u^{2}$ belongs $C N D(X, \sigma)$. Consequently, so does $h_{1}^{b}$, for $b \in(0,1]$. Thus, Assertion $(i i i)$ follows from $(i i)$.

In the last two results in the paper, we will employ the previous results in order to obtain positive definite functions on $S^{m} \times X$, where $X$ is a quasi-metric space.

Theorem 3.3. Let $m$ be a positive integer. Let $G_{\alpha}$ be the function defined at Equation (1.2) with $f$ being bounded and completely monotone. Then, the following assertions hold:

(i) $G_{\alpha}$ belongs to $P D\left(S^{m} \times X,\left(2-2 \cos \theta_{m}\right)^{a}, \sigma\right)$ provided $(X, \sigma)$ is a quasi-metric space, $h$ is a nonnegative valued function in $C N D(X, \sigma), \alpha \geq(m+1) / 2$ and $a \in(0,1]$;

(ii) $G_{\alpha}$ belongs to $P D\left(S^{m} \times S^{l},\left(2-2 \cos \theta_{m}\right)^{a}, \theta_{l}^{b}\right)$ provided $l \geq 1, \alpha \geq(m+1) / 2, a, b \in(0,1]$ and $h$ is a Bernstein function.

Proof. Assertion $(i)$ follows from the obvious identity

$$
\|x-y\|^{2}=2-2 \cos \theta_{m}(x, y), \quad x, y \in S^{m},
$$

in concert with Theorem 3.2- $(i)$. As for Assertion $(i i)$, we first notice that $\left(S^{l}, \theta_{l}^{1 / 2}\right)$ is isometrically embeddable in $\left(S^{\infty}, \theta_{\infty}^{1 / 2}\right)$. On the other hand, arguments in [24] show that $\left(S^{\infty}, \theta_{\infty}^{1 / 2}\right)$ is isometrically embeddable in a Hilbert space. Thus, the assertion follows from Assertion $(i)$ and Theorem 3.2-(iii). 
It becomes natural to ask whether Theorem 3.3 still holds when the metric $\left(2-2 \cos \theta_{m}\right)^{a}$ is replaced with the geodesic $\theta_{m}$. The answer seems to rely on a suitable choice of the completely monotone function $f$ in the definition of $G_{\alpha}$. Below, we show that it is true whenever $\alpha, s>0$, $f(t)=(s+t)^{-\alpha}$ and $h$ belongs $C N D(X, \sigma)$. Indeed, it suffices to observe that

$$
\frac{\Gamma(\alpha)}{(t+\operatorname{sh}(u))^{\alpha}}=\int_{0}^{\infty} e^{-t x} e^{-s h(u) x} x^{\alpha-1} d x .
$$

Applying Proposition 2.3 once again, it is now seen that

$$
(t, u) \in[0, \pi] \times D_{X}^{\sigma} \mapsto \frac{\Gamma(\alpha)}{(t+\operatorname{sh}(u))^{\alpha}}
$$

belongs to $P D\left(S^{m} \times X, \theta_{m}, \sigma\right)$. Finally, one needs to observe that

$$
\frac{1}{(t+\operatorname{sh}(u))^{\alpha}}=\frac{1}{h(u)^{\alpha}} f\left(\frac{t}{h(u)}\right)=G_{\alpha}(t, u), \quad(t, u) \in[0, \pi] \times D_{X}^{\sigma} .
$$

The elaborations above suggest that a special class of completely monotonic functions might turn to be useful for the result that follows. Following [12], we call a function $f:[0, \infty) \rightarrow \mathbb{R}$ a generalized Stieltjes function of order $\lambda$ if

$$
f(x)=C+\int_{0}^{\infty} e^{-x r} r^{\lambda-1} \phi(r) d r
$$

for some completely monotone function $\phi$ and some $C \geq 0$.

Theorem 3.4. Let $m$ be a positive integer and $(X, \sigma)$ a quasi-metric space. Let $G_{\alpha}$ be the function defined at Equation (1.2) with $f$ a generalized Stieltjes function of order $\lambda>0$. Then, $G_{\alpha}$ belongs to $P D\left(S^{m} \times X, \theta_{m}, \sigma\right)$ provided $\alpha \geq \lambda$ and $h$ is a nonnegative valued function in $C N D(X, \sigma)$.

Proof. According to [20, p. 16], we can write

$$
\begin{aligned}
G_{\epsilon+\lambda}(t, u) & =\frac{1}{h(u)^{\epsilon+\lambda}}\left[A+\int_{0}^{\infty} \frac{h(u)^{\lambda}}{(r h(u)+t)^{\lambda}} d r\right] \\
& =\frac{A}{h(u)^{\epsilon+\lambda}}+\frac{1}{h(u)^{\epsilon}} \int_{0}^{\infty} \frac{1}{(r h(u)+t)^{\lambda}} d r, \quad t \geq 0 ; u \in D_{X}^{\sigma} ; \epsilon \geq 0,
\end{aligned}
$$

for some positive constant $A$ and a convenient positive measure $\mu$ on $[0, \infty)$. Since the function in Equation (3.7) belongs to $P D\left(S^{m} \times X, \theta_{m}, \sigma\right)$, when $h$ is a nonnegative valued function in $C N D(X, \sigma)$, the same is true for

$$
(t, u) \in[0, \pi] \times D_{X}^{\sigma} \mapsto \int_{0}^{\infty} \frac{1}{(r h(u)+t)^{\lambda}} d r .
$$

Invoking Proposition 2.3 and taking into account that the class $P D\left(S^{m} \times X, \theta_{m}, \sigma\right)$ is a convex cone, it follows that $(t, u) \in[0, \pi] \times D_{X}^{\sigma} \mapsto G_{\epsilon+\lambda}(t, u)$ belongs to $P D\left(S^{m} \times X, \theta_{m}, \sigma\right)$. The proof is completed.

Deeper results providing generalizations of Gneiting's result via generalized Stieltjes functions were obtained recently in [13]. In particular, it provides concrete examples of completely monotone functions $f$ that lead to classes $\left\{G_{\alpha}: \alpha>0\right\}$ of strictly positive definite functions.

Acknowledgment. The authors would like to thank two anonymous referees for several suggestions and corrections which led to an improved version of the paper. V. A. Menegatto 
acknowledges partial financial support from FAPESP, grant 2016/09906-0. E. Porcu acknowledges the support of grant CONICYT/FONDECYT/REGULAR/No. 1170290 from the Chilean Commission for Scientific and Technological Research.

\section{REFERENCES}

[1] N. I. Akhiezer: Lectures on Integral transforms. Translated from Russian by H. H. McFaden. Translations of Mathematical Monographs, 70. American Mathematical Society, Providence, RI, 1988.

[2] T. Apanasovich, M. Genton: Cross-covariance functions for multivariate random fields based on latent dimensions. Biometrika 97 (2010), 15-30.

[3] A. Belton, D. Guillot, A. Khare, and M. Putinar: A Panorama of Positivity I: Dimension Free. In: Aleman A., Hedenmalm H., Khavinson D., Putinar M. (eds) Analysis of Operators on Function Spaces. Trends in Mathematics. Birkhäuser, Cham, 2019.

[4] C. Berg, E. Porcu: From Schoenberg coefficients to Schoenberg functions. Constr. Approx. 45 (2017), 217-241.

[5] D. J. Daley, E. Porcu: Dimension walks and Schoenberg spectral measures. Proc. Amer. Math. Soc. 141 (2013), 18131824.

[6] T. Fonseca, M. Steel: A general class of nonseparable space-time covariance models. Environmetrics 22 (2011), 224-242.

[7] T. Gneiting: Nonseparable, stationary covariance functions for space-time data. J. Amer. Statist. Assoc. 97 (2002), 590600.

[8] T. Gneiting, M. Genton and P. Guttorp: Geostatistical space-time models, stationarity, separability and full symmetry. Finkenstaedt, B., Held, L. and Isham, V. (eds.), Statistics of Spatio-Temporal Systems, Chapman \& Hall/CRC Press, pp. 151-175, 2007.

[9] I. S. Gradshteyn, I. Ryzhik: Table of integrals, series, and products. Fourth edition prepared by Ju. V. Geronimus and M. Ju. Ceitlin. Translated from Russian by Scripta Technica, Inc. Translation edited by Alan Jeffrey Academic Press, New York-London, 1965.

[10] J. C. Guella, V. A. Menegatto: Schoenberg's theorem for positive definite functions on products: a unifying framework. J. Fourier Anal. Appl. 25 (2019), 1424-1446.

[11] R. Horn, C. Johnson: Topics in matrix analysis. Corrected reprint of the 1991 original. Cambridge University Press, Cambridge, 1994.

[12] D. Karp, E. Prilepkina: Generalized Stieltjes functions and their exact order. J. Class. Anal. 1 (2012), 143-152.

[13] V. A. Menegatto: Positive definite functions on products of metric spaces via generalized Stieltjes functions, Proc. Amer. Math. Soc (2020), to appear.

[14] V. A. Menegatto: Strictly positive definite kernels on the Hilbert sphere. Appl. Anal. 55 (1994), 91-101.

[15] E. Porcu, M. Bevilacqua and M. Genton: Spatio-temporal covariance and cross-covariance functions of the great circle distance on a sphere. J. Amer. Stat. Assoc. 97 (2016), 590-600.

[16] E. Porcu, P. Gregori and J. Mateu: Nonseparable stationary anisotropic space-time covariance functions. Stoch. Environ. Res. Risk Assess. 21 (2006), 113-122.

[17] E. Porcu, J. Mateu: Mixture-based modeling for space-time data. Environmetrics 18 (2007), 285-302.

[18] E. Porcu, J. Mateu and G. Christakos: Quasi-arithmetic means of covariance functions with potential applications to space-time data. J. Multivariate Anal. 100 (2009), 1830-1844.

[19] A. Poularikas: The handbook of formulas and tables for signal processing. CRC Press, Boca Ratón, 1999.

[20] R. L. Schilling, R. Song and Z. Vondracek: Bernstein functions. Theory and applications. Second edition. De Gruyter Studies in Mathematics, 37. Walter de Gruyter \& Co., Berlin, 2012.

[21] M. Schlather: Some covariance models based on normal scale mixtures. Bernoulli 16 (2010), 780-797.

[22] I. J. Schoenberg: Metric spaces and completely monotone functions. Ann. of Math. 39 (1938), 811-841.

[23] I. J. Schoenberg: Metric spaces and positive definite functions. Trans. Amer. Math. Soc. 44 (1938), 522-536.

[24] I. J. Schoenberg: Positive definite functions on spheres. Duke Math. J. 9 (1942), 96-108.

[25] K. Triméche: Generalized harmonic analysis and wavelet packets. Gordon and Breach Science Publishers, 2001.

[26] D. Widder: The Laplace Transform. Princeton University Press, Princeton, 1966.

[27] P. White, E. Porcu: Towards a complete picture of covariance functions on spheres cross time. Electron. J. Stat. 13 (2019), 2566-2594.

[28] J. H. Wells, L. R. Williams: Embeddings and extensions in analysis. Ergebnisse der Mathematik und ihrer Grenzgebiete, Band 84. Springer-Verlag, New York-Heidelberg, 1975.

[29] H. Wendland: Scattered data approximation. Cambridge Monographs on Applied and Computational Mathematics Volume 17, Cambridge University Press, 2001.

[30] V. Zastavnyi, E. Porcu: Characterization theorems for the Gneiting class of space-time covariances. Bernoulli 17 (2011), 456-465. 
ICMC-UniversidAde DE SÃo PAUlo, BRAZIL

ORCID: 0000-0002-4213-8759

E-mail address: menegatt@icmc.usp.br

IMC-Universidade Federal de ItAJUbÁ, BRAZIL

ORCID: 0000-0002-6079-2236

E-mail address: oliveira@unifei.edu.br

School of Computer Science and Statistics, Trinity College, Dublin, Ireland ORCID: 0000-0001-9934-4409

E-mail address: georgepolya01@gmail.com 